\title{
PETS 2015: datasets and challenge
}

Conference or Workshop Item

Accepted Version

Li, L., Nawaz, T. and Ferryman, J. (2015) PETS 2015:

datasets and challenge. In: 12th IEEE International

Conference on Advanced Video- and Signal-based

Surveillance (AVSS2015), August 25-28, 2015, pp. 1-6.

Available at http://centaur.reading.ac.uk/46540/

It is advisable to refer to the publisher's version if you intend to cite from the work. See Guidance on citing.

Published version at: http://ieeexplore.ieee.org/xpl/articleDetails.jsp?arnumber $=7301741$

All outputs in CentAUR are protected by Intellectual Property Rights law, including copyright law. Copyright and IPR is retained by the creators or other copyright holders. Terms and conditions for use of this material are defined in the End User Agreement.

www.reading.ac.uk/centaur 


\section{CentAUR}

Central Archive at the University of Reading

Reading's research outputs online 


\title{
PETS 2015: Datasets and Challenge
}

\author{
Longzhen Li, Tahir Nawaz and James Ferryman \\ Computational Vision Group, School of Systems Engineering, University of Reading, UK \\ \{longzhen.li | t.h.nawaz | j.m.ferryman \}ereading.ac.uk
}

\begin{abstract}
This paper presents the two datasets (ARENA and P5) and the challenge that form a part of the PETS 2015 workshop. The datasets consist of scenarios recorded by using multiple visual and thermal sensors. The scenarios in ARENA dataset involve different staged activities around a parked vehicle in a parking lot in $U K$ and those in P5 dataset involve different staged activities around the perimeter of a nuclear power plant in Sweden. The scenarios of each dataset are grouped into 'Normal', 'Warning' and 'Alarm' categories. The Challenge specifically includes tasks that account for different steps in a video understanding system: Low-Level Video Analysis (object detection and tracking), Mid-Level Video Analysis ('atomic' event detection) and High-Level Video Analysis ('complex' event detection). The evaluation methodology used for the Challenge includes well-established measures.
\end{abstract}

\section{Introduction}

Video surveillance is a widely-researched field presently. Several techniques have been designed and tested for the tasks of object detection and tracking as well as for detection of events of interest. However it is still difficult to compare or evaluate such algorithms because of the lack of standard metrics and benchmarks that indicate how detection, tracking and threat analysis system perform against a common database. The goal of the PETS workshop has been to foster the emergence of computer vision technologies for detection and tracking by providing evaluation datasets and metrics that allow an accurate assessment and comparison of such methodologies. PETS 2015 is sponsored by the EU project P5, the Privacy Preserving Perimeter Protection Project, that aims to develop an intelligent perimeter proactive surveillance system that works robustly under a wide range of weather and lighting condi-

This project has received funding from the European Union's Seventh Framework Programme for research, technological development and demonstration under grant agreement no. 312784. tions for the protection of critical infrastructures ${ }^{1}$.

PETS 2015 workshop includes a Challenge and provides two datasets (ARENA and P5) to enable the community to test and rank the algorithms on $^{2}$.The ARENA dataset includes a selection of video sequences from PETS 2014 dataset [4] that are made available by another EU project ARENA $^{3}$, which addresses the design of a flexible surveillance system to enable situational awareness and determination of potential threats on mobile assets in transit. The P5 dataset contains multi-modal, multi-sensor recordings involving different staged activities around the perimeter of the OKG nuclear plant outside Oskarshamn, Sweden and was recorded jointly by the P5 project partners. Overall, the two datasets cover a variety of tasks (to constitute the PETS 2015 Challenge) involving low-level video analysis (object detection and tracking), mid-level analysis ('atomic' or simple event detection) and high-level analysis (complex 'threat' event detection).

The remainder of this paper is organised as follows. Section 2 presents the PETS 2015 datasets in detail. The PETS 2015 Challenge is described in Section 3. The evaluation framework used in the Challenge is described in detail in Section 4. Section 5 concludes this paper.

\section{Datasets}

PETS 2015 consists of two datasets:

- ARENA dataset: a multi-sensor dataset as used for the PETS2014 challenge which addresses protection of critical mobile assets.

- P5 dataset: a multi-modal multi-sensor dataset addressing the application of multi sensor surveillance to protect a nuclear power plant.

The ARENA dataset was used in its full form in PETS 2014 Challenge [4]. For PETS 2015, a selection of the ARENA dataset is used by including fewer scenarios that are more relevant to PETS 2015 Challenge. The selected

\footnotetext{
${ }^{1}$ http://www.p5-fp7.eu

${ }^{2}$ http://pets2015.net

${ }^{3}$ http://www.arena-fp7.eu
} 
Table 1. Sensor properties for ARENA dataset

\begin{tabular}{|c|c|c|c|}
\hline ID & Model & Resolution (pxl) & Frame Rate \\
\hline \hline ENV_RGB_3 & PTZ Axis 233D & $768 \times 576$ & 7 \\
\hline TRK_RGB_1 & Basler BIP2-1300c-dn & $1280 \times 960$ & 30 \\
\hline TRK_RGB_2 & Basler BIP2-1300c-dn & $1280 \times 960$ & 30 \\
\hline
\end{tabular}

scenarios from ARENA and P5 datasets are grouped into 'Normal', 'Warning' and 'Alarm' categories. 'Normal' alludes to activities that do not pose any threat. 'Warning' refers to abnormal activities that may potentially develop into a threat. 'Alarm' refers to activities that cause a threat in the scene and hence require immediate action. A detailed description of the two datasets are given below.

\subsection{ARENA dataset}

\subsubsection{Overview}

The ARENA dataset comprises of a series of multi-camera video recordings where the main subject is the detection and understanding of human behaviour around a parked vehicle. The main objective is to detect and understand the different behaviours from visual (RGB) cameras mounted on the vehicle itself. With this dataset already available for download from PETS 2014 workshop, PETS 2015 workshop provides the opportunity for researchers and industry to submit methodological advances and results obtained using this dataset since the 2014 workshop.

\subsubsection{Camera setup and characteristics}

Environmental camera: One visual camera ENV_RGB_3 is used (Table 1) that is installed at the location as shown in Figure 1 to cover an approximate area of $100 \mathrm{~m} \times 30 \mathrm{~m}$. This camera provides a global view of the monitored area.

On-board cameras. Originally four non-overlapping visual cameras were mounted at each corner of a truck in

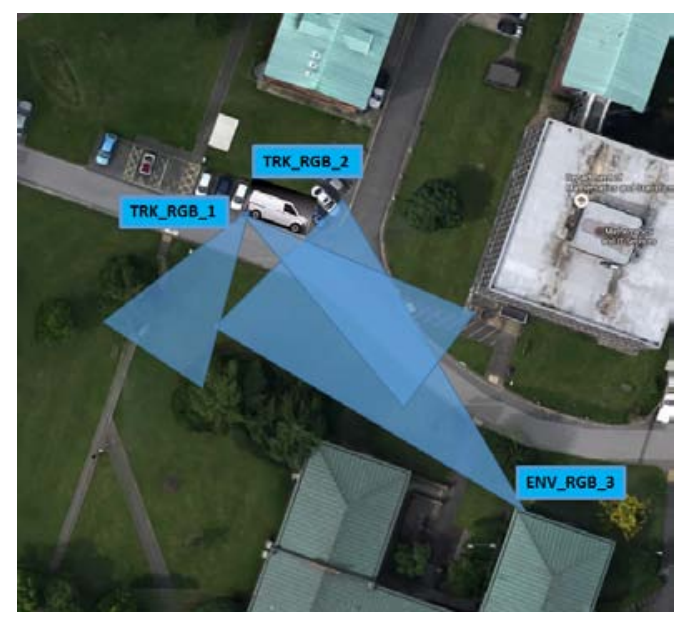

Figure 1. Sensor locations and their FOVs for ARENA dataset
ARENA dataset. The selective part of ARENA dataset used for PETS 2015 Challenge includes two on-board cameras (TRK_RGB_1, TRK_RGB_2) at the locations shown in Figure 1. Table 1 lists the sensors while describing their respective characteristics.

\subsubsection{Scenarios}

The dataset scenarios ('Normal', 'Warning', 'Alarm') are listed in Table 2.

\subsection{P5 dataset}

\subsubsection{Overview}

The dataset contains sequences with different activities staged around the perimeter of the OKG nuclear plant outside Oskarshamn, Sweden. The dataset was recorded by P5 partners collectively by using multiple types of surveillance sensors including digital IP cameras and thermal sensors.

\subsubsection{Camera setup and characteristics}

There are five visual and thermal sensor positions covering a large area with $550 \mathrm{~m}$ from one end to the other on the land side (see Figure 2). It takes 10-15 minutes to walk from one end to the other.

Visible sensors. Three visual cameras (VS_1, VS_2, VS_3) at the locations shown in Figure 2 are selected to mainly cover the road along the water area. Most of the scenarios take place in the monitored region.

Thermal sensors. Two of the thermal sensors (TH_3, TH_4) as shown in Figure 2 are installed side by side with visual cameras, with the aim to provide similar Field of Views (FOVs) to that of visual cameras. The main benefit of the joint use of thermal and visible sensors is that different modalities provide complementary information of the scene captured by thermal infrared spectrum and visible light spectrum respectively. Two more thermal sensors TH_1 and TH_2 are installed at the locations shown in Figure 2 , which mainly cover the long road along the fence outside the nuclear plant.

The sensor properties are summarised in Table 3.

\subsubsection{Scenarios}

The dataset scenarios ('Normal', 'Warning', 'Alarm') are listed in Table 4.

Sample images from the cameras for both ARENA and P5 datasets are shown in Figure 3.

\section{Challenge}

The PETS 2015 Challenge addresses the application of automated sensor surveillance for the protection of critical 
Table 2. List and description of scenarios for ARENA dataset

\begin{tabular}{|c|c|l|c|}
\hline Scenario type & ID & \multicolumn{1}{|c|}{ Description } & Challenges \\
\hline \hline Normal & N1_ARENA & Persons walking in a group & Scale change; occlusion; pose change \\
\hline Warning & W1_ARENA & Driver falls after being hit by someone & Occlusion; scale change; person running \\
\hline \multirow{2}{*}{ Alarm } & A1_ARENA & Driver involved in a fight with someone & Scale change; pose change; occlusion; clutter \\
\cline { 2 - 4 } & A2_ARENA & Driver attacked by someone from a car & Scale change; speed change; occlusion \\
\hline
\end{tabular}

infrastructure. It aims to bring together researchers, practitioners and students from computer vision and surveillancerelated fields to share knowledge on methodologies, features and results related to the evaluation, modelling and understanding of object motion and behaviour from video analytics.

Submissions are solicited that either:

- Describe an approach to low-level video analysis / mid-level video analysis / high-level video analysis (see below) and report results based on the datasets provided for this workshop. The actual results are also to be submitted in XML format which will be described in Section 4.2.

- Contribute to general performance evaluation methodology for detection, tracking and behaviour (threat) analysis. It is not necessary to explicitly consider the datasets provided for the workshop, however one is encouraged to use the PETS datasets that are made available.

The PETS 2015 datasets (i.e. ARENA dataset and P5 dataset) are designed to accommodate different categories in a typical video surveillance system: Low-level video analysis, Mid-level video analysis and High-level video analysis. Within each category, one or more specific vision tasks are further defined to address the diversity of the challenges as well as different level of complexity. These tasks are described next.

\section{Object tracking}

The task involves detecting and/or tracking objects in

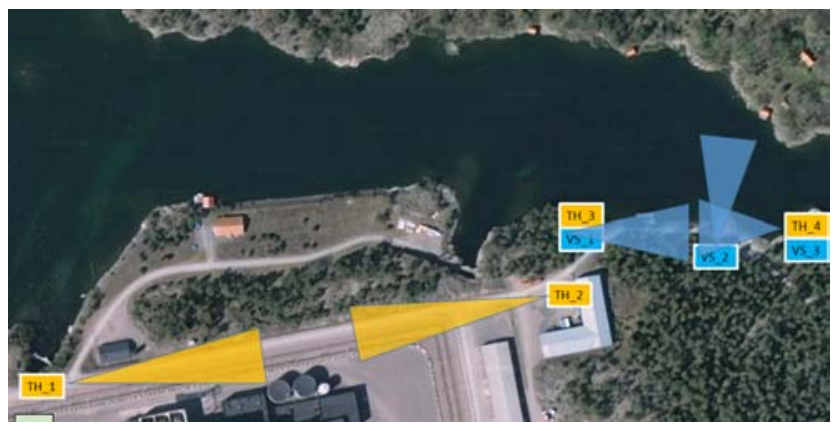

Figure 2. Sensor locations and their FOVs for P5 dataset all frames of the video sequences specified for this task.

\section{'Group walking' event detection}

The task involves detection of the occurrence of the event of a group of people walking in a segment of the specified video sequences. A group is defined to consist of more than two people walking together.

\section{'Person running' event detection}

The task involves detection of the occurrence of the event of a person running in a segment of the specified video sequences.

\section{'Threat' event detection}

The task involves detection of the occurrence of a threat event in a segment of the specified video sequences. A threat event generally consists of a sequence of simpler atomic events.

The paper submission for PETS 2015 Challenge can take place for any (one or more) of the above tasks using ARENA and/or P5 datasets. The PETS 2015 Challenge indeed focuses on single-camera processing only. This means for a task, a participant must run its algorithm independently on each of the corresponding single-camera video sequences specified for this task. All sequences under each task for each category are listed in Table 5.

\section{Evaluation Methodology}

\subsection{Ground truth}

To enable a precise quantitative comparison and ranking of various algorithms, efforts are made to provide accurate

Table 3. Sensor properties for P5 dataset (VS: Visual, TH: Thermal)

\begin{tabular}{|c|c|c|c|}
\hline ID & Model & Resolution (pxl) & Frame Rate \\
\hline \hline VS_1 & Basler BIP2-1300c-dn & $1280 \times 960$ & 25 \\
\hline VS_2 & Basler BIP2-1300c-dn & $1280 \times 960$ & 15 \\
\hline VS_3 & Basler BIP2-1300c-dn & $1280 \times 960$ & 25 \\
\hline TH_1 & FLIR SC655 & $640 \times 480$ & 25 \\
\hline TH_2 & FLIR SC655 & $640 \times 480$ & 12.5 \\
\hline TH_3 & FLIR SC655 & $640 \times 480$ & 25 \\
\hline TH_4 & FLIR A65 & $640 \times 512$ & 30 \\
\hline
\end{tabular}


Table 4. List and description of scenarios for P5 dataset

\begin{tabular}{|c|c|c|c|}
\hline Scenario type & ID & Description & Challenges \\
\hline \hline Normal & N1_P5 & A vehicle driving across the scene & Scale change; pose change; speed change; clutter \\
\hline Warning & W1_P5 & A group of 6 people walking across the scene & Occlusion; scale change; clutter; speed change \\
\hline Alarm & A1_P5 & An abandoned bag is picked up suspiciously & Scale change; pose change; clutter; speed change \\
\hline
\end{tabular}

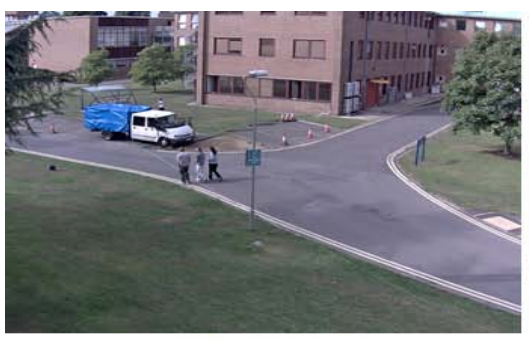

ENV_RGB_3

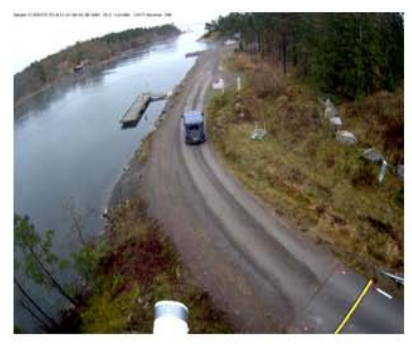

VS 1

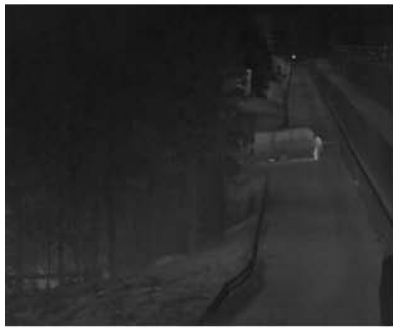

TH_1

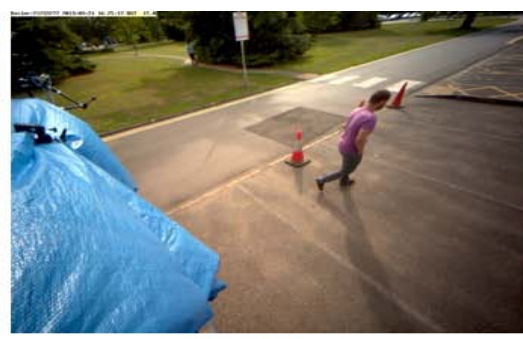

TRK_RGB_1

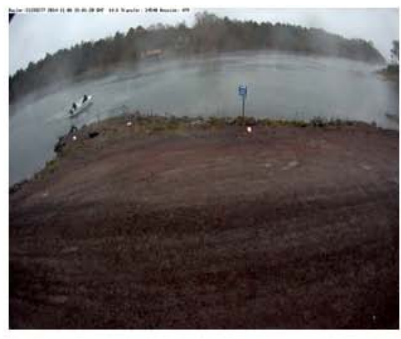

VS_2

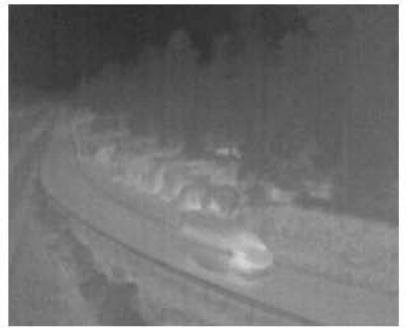

TH_2

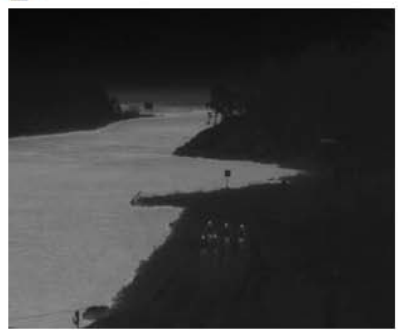

TH_3

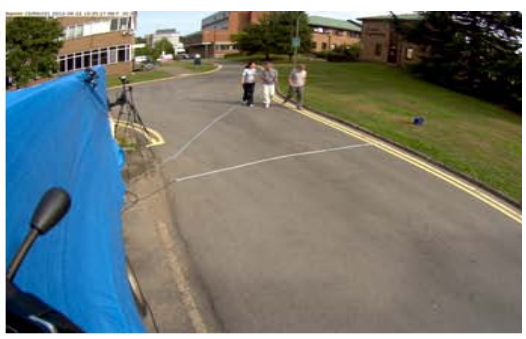

TRK_RGB_2

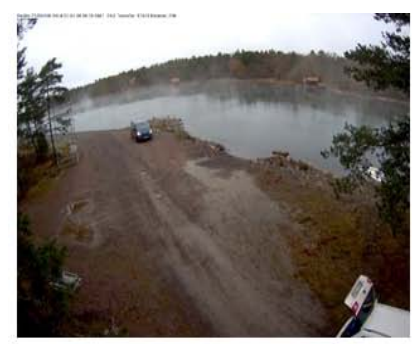

VS_3

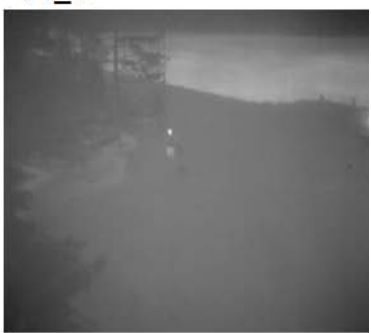

TH_4

Figure 3. Top row: sample images from ARENA dataset; middle row: sample images for visual sensors from P5 dataset; bottom row: sample images for thermal sensors from P5 dataset.

Table 5. List of sequences under each task for PETS 2015 Challenge

\begin{tabular}{|c|c|c|c|}
\hline Task & Category & Sequences (ARENA dataset) & Sequences (P5 dataset) \\
\hline Object tracking & Low-level analysis & $\begin{array}{c}\text { N1_ARENA-Tg_ENV_RGB_3; N1_ARENA-Tg_TRK_RGB_1; } \\
\text { N1_ARENA-Tg_TRK_RGB_2; W1_ARENA-Tg_ENV_RGB_3; } \\
\text { W1_ARENA-Tg_TRK_RGB_1; A1_ARENA-Tg_ENV_RGB_3; } \\
\text { A1_ARENA-Tg_TRK_RGB_2 }\end{array}$ & $\begin{array}{l}\text { N1_P5-Tg_VS_1; N1_P5-Tg_VS_3; } \\
\text { N1_P5-Tg_TH_1; N1_P5-Tg_TH_2; } \\
\text { W1_P5-Tg_VS_1; W1_P5-Tg_VS_3; } \\
\text { W1_P5-Tg_TH_3; A1_P5-Tg_VS_2; } \\
\text { A1_P5-Tg_TH_3 }\end{array}$ \\
\hline $\begin{array}{l}\text { 'Group walking' } \\
\text { event detection }\end{array}$ & Mid-level analysis & $\begin{array}{c}\text { N1_ARENA-Gp_ENV_RGB_3; N1_ARENA-Gp_TRK_RGB_1; } \\
\text { N1_ARENA_Gp_TRK_RGB_2 }\end{array}$ & $\begin{array}{l}\text { W1_P5-Gp_VS_1; W1_P5-Gp_VS_3; } \\
\text { W1_P5-Gp_TH_3 }\end{array}$ \\
\hline $\begin{array}{l}\text { 'Person running' } \\
\text { event detection }\end{array}$ & Mid-level analysis & $\begin{array}{c}\text { W1_ARENA-Rg_ENV_RGB_3; W1_ARENA-Rg_TRK_RGB_1; } \\
\text { A2_ARENA-Rg_ENV_RGB_3 }\end{array}$ & $\begin{array}{c}\text { W1_P5-Rp_VS_1; W1_P5-Rp_VS_3; } \\
\text { W1_P5-Rp_TH_3 }\end{array}$ \\
\hline $\begin{array}{l}\text { 'Threat' } \\
\text { event detection }\end{array}$ & High-level analysis & $\begin{array}{l}\text { A1_ARENA-Tt_ENV_RGB_3; A1_ARENA-Tt_TRK_RGB_2 } \\
\text { A2_ARENA-Tt_ENV_RGB_3; A2_ARENA-Tt_TRK_RGB_2 }\end{array}$ & $\begin{array}{c}\text { A1_P5-Tt_VS_2; A1_P5-Tt_TH_3 } \\
\text { A1_P5-Tt_TH_4 }\end{array}$ \\
\hline
\end{tabular}




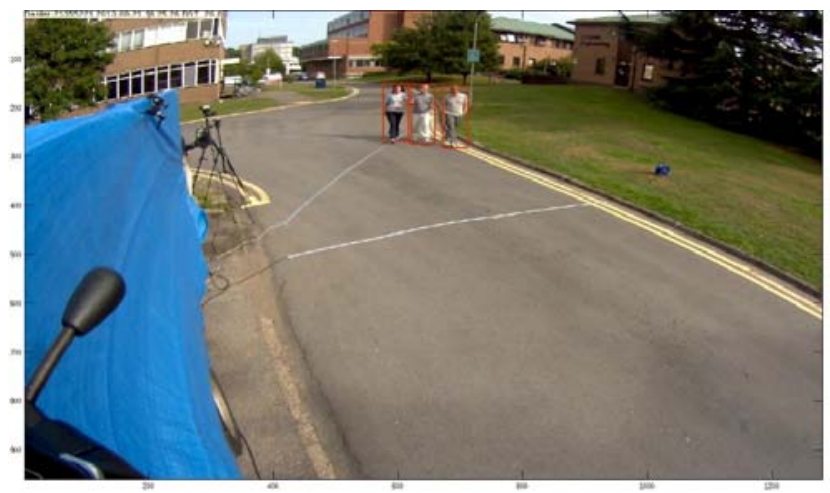

Figure 4. Object tracking annotation: A sample annotated image for a camera view with a red bounding box overlaid around each object.

and detailed annotations for PETS 2015 datasets. Effectively two types of ground truth annotation are obtained, corresponding to object tracking and event detection tasks as discussed in Section 3. The annotation process and format are describe in detail in the following sub sections.

\subsubsection{Object tracking}

For the Object Tracking task, the aim is to detect and track objects in all frames of the video sequences specified for this task. Consequently, the ground truth is abtained for every single frame of all the sequences within this task. The annotation is obtained in the format of bounding box which effectively encloses each object in each frame. Figure 4 shows a sample annotated image for a camera view with red bounding boxes overlaid around each object. Note in the case of occlusion, only the visible part of the object is annotated. Table 6 presents a summary for the annotation

Table 6. Annotation summary for object tracking task

\begin{tabular}{|c|c|c|c|}
\hline Sequence & \# Frames & \# Object & Object Type \\
\hline \hline N1_P5-VS_1 & 400 & 1 & Vehicle \\
\hline N1_P5-VS_3 & 387 & 1 & Vehicle \\
\hline N1_P5-TH_1 & 600 & 1 & Vehicle \\
\hline N1_P5-TH_2 & 220 & 1 & Vehicle \\
\hline W1_P5-VS_1 & 180 & 6 & Person \\
\hline W1_P5-VS_3 & 180 & 6 & Person \\
\hline W1_P5-TH_3 & 740 & 6 & Person \\
\hline A1_P5-VS_2 & 720 & 1 & Boat \\
\hline A1_P5-TH_3 & 1000 & 1 & Boat \\
\hline N1_ARENA-Tg_ENV_RGB_3 & 289 & 5 & Person \\
\hline N1_ARENA-Tg_TRK_RGB_1 & 513 & 5 & Person \\
\hline N1_ARENA-Tg_TRK_RGB_2 & 684 & 5 & Person \\
\hline W1_ARENA-Tg_ENV_RGB_3 & 155 & 3 & Person \\
\hline W1_ARENA-Tg_TRK_RGB_1 & 240 & 3 & Person \\
\hline A1_ARENA-Tg_ENV_RGB_3 & 295 & 4 & Person \\
\hline A1_ARENA-Tg_TRK_RGB_2 & 670 & 4 & Person \\
\hline
\end{tabular}

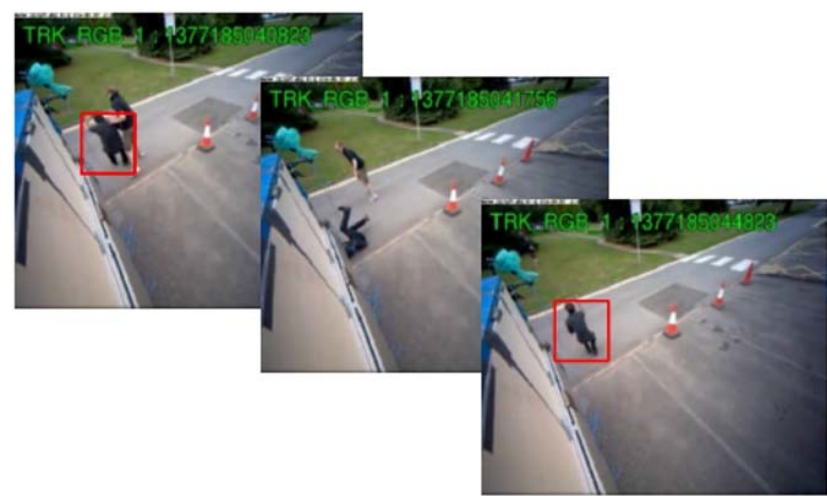

Figure 5. Event detection annotation: An example with start and final frame numbers of an event annotated and the bounding box overlaid around the object under consideration in the two frames.

task performed for object tracking.

\subsubsection{Event detection}

For the event detection task, the event is annotated by defining the start and end frame of the event, as well as the bounding box(es) for the main target(s) involved in the event. An example of the annotation of an event detection annotation is illustrated in Figure 5, with a red box overlaid around the person in the start and final frames of the defined event.

\subsection{Submission format}

Collectively researchers develop their algorithms on different computing platforms, use a variety of programming languages and typically store their algorithm results in their own data structures. A standard file format for the submission of results is therefore required in order to evaluate different researchers' methods. For PETS 2015 workshop, a requirement was set that submitted papers were accompanied with algorithmic results in XML format. This way, the object detection and tracking results can be reconstructed from the XML files, which provided for a qualitative comparison of a number of algorithms operating on the same PETS video sequences.

The submitted XML file must conform to the PETS 2015 XML Schema which can be found on the PETS 2015 website [5]. An XML Schema is a definition on how to construct a valid XML file.

\subsection{Evaluation metrics}

Object tracking: Tracking evaluation accounts for the three key aspects including tracking accuracy (extent of match between an estimation and the corresponding ground truth), cardinality error (difference between the number of 
estimated targets and the number of ground-truth targets) and ID change (wrong associations between estimated and ground-truth targets) [3]. For the Challenge, the widelyused Multiple Object Tracking Accuracy (MOTA) [2] is used, which takes into account the cardinality error (in the form of false positives and false negatives) and ID changes without explicitly considering accuracy. MOTA is defined as follows:

$$
\operatorname{MOTA}=1-\frac{\sum_{k=1}^{K}\left(c_{1}\left|F N_{k}\right|+c_{2}\left|F P_{k}\right|+c_{3}\left|I D C_{k}\right|\right)}{\sum_{k=1}^{K} v_{k}},
$$

where the parameters $c_{1}, c_{2}$ and $c_{3}$ determine the contributions from the number of false negatives $\left(\left|F N_{k}\right|\right)$, number of false positives $\left(\left|F P_{k}\right|\right)$ and number of ID changes $\left(\left|I D C_{k}\right|\right)$ at a frame $k$, respectively, and $v_{k}$ is the number of ground-truth targets at frame $k . c_{1}=1, c_{2}=1, c_{3}=\log _{10}$ as described in the paper [2]. False negatives are the missed targets at frame $k$ and false positives are the estimated targets with overlap $O_{k, t}<\bar{\tau}$ such that $\bar{\tau}$ is a pre-defined threshold and $O_{k, t}=\frac{\left|\bar{A}_{k, t} \cap A_{k, t}\right|}{\left|A_{k, t} \cup A_{k, t}\right|}$ for a $t$ th pair of groundtruth and estimated bounding boxes at frame $k . \bar{A}_{k, t}$ and $A_{k, t}$ denote the occupied regions on the image plane for the ground-truth and estimated bounding boxes, respectively. $\bar{\tau}$ is often set to 0.5 [1]. MOTA $\leq 1$ : the higher MOTA, the better the performance. To evaluate tracking accuracy, a recently-introduced measure, Multiple Extendedtarget Lost-Track ratio (MELT) [3], is used. MELT provides accuracy evaluation using the information about losttrack ratio. Let $N_{i}$ be the total number of frames in the $i$ th ground-truth track and $N_{i}^{\tau}$ is the number of frames with the overlap score below a threshold $\tau$, then the lost-track ratio $\lambda_{i}^{\tau}$ is computed as follows: $\lambda_{i}^{\tau}=\frac{N_{i}^{\tau}}{N_{i}}$. MELT for a particu$\operatorname{lar} \tau$ is computed as follows: $\operatorname{MELT}_{\tau}=\frac{1}{V} \sum_{i=1}^{V} \lambda_{i}^{\tau}$, where $V$ is the total number of ground-truth tracks, and

$$
\text { MELT }=\frac{1}{S} \sum_{\tau \in[0,1]} \operatorname{MELT}_{\tau},
$$

provides the overall tracking accuracy for a full variation of $\tau$, where $S$ is the number of sampled values of $\tau$. MELT $\in$ $[0,1]$ : the lower the value the better the performance.

Event detection: Let $K_{i n i}^{\text {est }}$ and $K_{\text {end }}^{\text {est }}$ be start and final frames, respectively, for a detected event by a candidate algorithm in a sequence, and $K_{i n i}^{g t}$ and $K_{e n d}^{g t}$ be the corresponding ground-truth information for the start and final frames, respectively, of the same event. The event detection error, $E$, is then computed as a distance between the estimated and ground-truth information:

$$
E=\sqrt{\left(K_{i n i}^{e s t}-K_{i n i}^{g t}\right)^{2}+\left(K_{\text {end }}^{e s t}-K_{\text {end }}^{g t}\right)^{2}} .
$$

$E \geq 0$ : the lower the value, the better the performance.

\section{Conclusions}

In this paper, we have described the datasets and the Challenge that are part of PETS 2015 workshop. Two datasets are presented that are the ARENA and P5 datasets. Each dataset is divided into three categories: Normal, Warning and Alarm. The ARENA dataset contains visible imagery recorded from multiple sensors installed onboard a critical asset (a truck) or in the environment. The P5 dataset contains both visible and thermal imagery recorded from multiple sensors installed outside the perimeter of a critical infrastructure (a nuclear power plant). The datasets account for the key challenges that are of interest within the community. The Challenge includes different surveillance tasks including object tracking, group walking event detection, person running event detection and threat event detection. The Challenge allows participants to run their algorithms for the specified tasks using the sequences provided under two datasets, and to submit the results that are then evaluated using the well established measures.

\section{References}

[1] B. Benfold and I. Reid. Stable multi-target tracking in realtime surveillance video. In Proc. of IEEE CVPR, pages $3457-$ 3464, 2011.

[2] R. Kasturi, D. Goldgof, P. Soundararajan, V. Manohar, J. Garofolo, R. Bowers, M. Boonstra, V. Korzhova, and J. Zhang. Framework for performance evaluation of face, text, and vehicle detection and tracking in video: Data, metrics, and protocol. IEEE TPAMI, 31(2):319-336, 2009.

[3] T. Nawaz, F. Poiesi, and A. Cavallaro. Measures of effective video tracking. IEEE TIP, 23(1):376-388, 2014.

[4] L. Patino and J. Ferryman. PETS 2014: Dataset and Challenge. In Proc. of IEEE Int. Conf. on AVSS, pages 355-360, Seoul, August 2014.

[5] IEEE International Workshop on Performance Evaluation of Tracking and Surveillance (PETS). http://pets2015.net/. 